Aspects of Data Quality in Psychology:

Missing Data and Aberrant Responses

Maxwell Hong, Matthew Carter, Casey Kim, Ying Cheng

University of Notre Dame

Correspondence concerning this article should be addressed to Ying Cheng, 390 Corbett Family Hall, Notre Dame, IN 46556, Email: ycheng4@nd.edu. This project is supported by the National Science Foundation grant SES-1853166. 


\begin{abstract}
Data preprocessing is an integral step prior to analyzing data in the social sciences. The purpose of this article is to report the current practices psychological researchers use to address data preprocessing or quality concerns with a focus on issues pertaining to aberrant responses and missing data in self report measures. 240 articles were sampled from four journals: Psychological Science, Journal of Personality and Social Psychology, Developmental Psychology, and Abnormal Psychology from 2012 to 2018. We found that nearly half of the studies did not report any missing data treatment $(111 / 240 ; 46.25 \%)$ and if they did, the most common approach to handle missing data was listwise deletion $(71 / 240 ; 29.6 \%)$. Studies that remove data due to missingness removed, on average, $12 \%$ of the sample. We also found that most studies do not report any methodology to address aberrant responses (194/240; 80.83\%). For studies that reported issues with aberrant responses, a study would classify $4 \%$ of the sample, on average, as suspect responses. These results suggest that most studies are either not transparent enough about their data preprocessing steps or maybe leveraging suboptimal procedures. We outline recommendations for researchers to improve the transparency and/or the data quality of their study.
\end{abstract} Keywords: Data quality, data preprocessing, missing data, aberrant responses, meta science 


\section{Aspects of Data Quality in Psychology: Missing Data and Aberrant Responses}

There have been several alarms raised about the methodological practices used in scientific research. A few major concerns include the large number of choices one has to make from designing a study and analyzing the data. This has been referred to in the statistical literature as the "garden of forking paths," where researchers may choose a path that corresponds to some desired outcome, such as statistical significance (Gelman and Loken, 2013). In psychology, the flexibility and number of choices are sometimes referred to as "researcher degrees of freedom" (Simmon, Nelson and Simonsohn, 2011). Through the choices one makes, researchers hope to achieve good quality data that can be used to answer their research questions. An example decision that every researcher has to make that pertains to data quality are exclusion criteria or data preprocessing. These choices start from the research design to the data analysis phase. They can have a significant impact on the findings from the study. For instance, removing participants for different reasons can vastly change the interpretation of a study. This study aims to investigate the practices researchers use to ensure good quality data for their study when using questionnaires.

There are many factors that affect data quality that could be addressed, or mitigated, through data preprocessing. This study will focus on two aspects that affect data quality: aberrant and missing data. Aberrant responders are individuals that deviate in some way from the majority of the sample (Hong, Steedle and Cheng, 2020). Aberrant responders may be classified as outliers; however, they also include individuals that respond too quickly to a task or fail to follow instructions (Meade and Craig, 2012), which do not represent natural variation in data but construct-irrelevant noise. Substantial amounts of missing data or certain patterns of missing data may be another indicator of poor data quality. One can examine the proportion of missing 
responses in a given data set. Meanwhile, the underlying cause for the missing response may be of interest (Peugh and Enders, 2004), which can influence the choice of the preprocessing method. Overall, the presence of aberrant or missing data can have deleterious effects on applied analysis where parameter estimates can be biased in unpredictable directions.

\section{Purpose of Study}

The confidence in results from psychological studies require methodologically informed decisions when choosing which path to take with missing or aberrant data. Several wrong turns may have an impact on the primary results. This study aims to summarize current practices related to data preprocessing in psychology. We conducted a systematic review in a diverse set of journals, Journal of Personality and Social Psychology, Developmental Psychology, Abnormal Psychology, and Psychological Science, in the years 2012 to 2018 . We report what type of methodology researchers utilize to ensure good data quality with a focus on aberrant responders and missing data. In order to keep the methodologies comparable across different domains of psychology, we focused on studies that used questionnaires. First, we review methods that can be used to improve data quality from both missing data and aberrant responders. We then describe the methods used to conduct a systematic review and the subsequent results. Finally, we provide recommendations for individuals to improve data quality from both a research design and statistical perspective.

\section{Data Quality}

\section{Missing Data}

We provide a brief review of missing data based on Rubin's (1976) theoretical

framework. For a detailed review, we refer readers to textbooks on the same topic (Enders, 2010; Rubin, 1976). Rubin outlined three types of missing data mechanisms that are useful to describe 
different missing data patterns. One type of missing data mechanism is missing completely at random (MCAR). This is where the missing values are unrelated to other observed variables in the data set as well as unobserved variables. The second case is missing at random (MAR). MAR considers the possibility that the missing values are related to other observed variables, but are not related to unobserved variables. The third and final case is missing not at random (MNAR). MNAR considers the possibility that a missing value is related to an unobserved variable. In each case, there are appropriate, and inappropriate, methodologies for missing data which depends on the underlying mechanism. Table 1 summarises the different missing data mechanisms and methodology

Table 1: Missing Data Mechanisms and Methodology Type of Missing Data Description Notes on Methodology

$\begin{array}{lll}\text { Missing Completely } & \text { Missing values are unrelated } & \text {. Many statistical methods } \\ \text { at Random } & \begin{array}{l}\text { to other observed variables in } \\ \text { the data set as well as }\end{array} & \text { provide unbiased estimates. } \\ & \text { unobserved variables. } & \text { Listwise or Pairwise } \\ & \text { deletion reduces power to } \\ \text { detect effects. }\end{array}$

Missing at Random Missing values are related to other observed variables, but are not related to unobserved variables.

Missing value is related to an unobserved variable
- Requires all variables related to missing data mechanisms.

- FIML and imputation provide unbiased estimates.

- All statistical methods provide biased estimates.

$\overline{\text { Note: This table was compiled based on works from Little and Rubin (2019) and Enders (2010). }}$ 
There have been several calls throughout academic history to consider proper handling of missing data. With Rubin's missing data mechanisms as a framework, there are implications for how each missing data method will perform. Listwise deletion removes all respondents who have missing values on at least one variable. There is also pairwise deletion, where data are removed on a case by case basis depending on the analysis. In general, both pairwise and listwise deletion result in lower statistical power under any missing data mechanism (Enders and Bandalos, 2001). Moreover, if the missing data come from MAR or MNAR, then a model's parameter estimates may be biased (Gomer and Yuan, in press).

Another method that is frequently recommended is imputation. There are several flavors of imputation, but the general gist is that the data are filled in with plausible values based on the observed data. For instance, there is mean imputation where the missing values are replaced by the mean of a variable. Mean imputation will produce biased estimates of any parameter except the mean, regardless of the missing data mechanism (Peugh and Enders, 2004). There is also regression-based imputation where missing values are replaced with predictions from a regression equation. Regression imputation can produce unbiased and consistent parameter estimates under MCAR or MAR (Little and Rubin, 2019). An advancement of regression imputation is multiple imputation. Multiple imputation takes into consideration the uncertainty of the imputed scores by fitting a regression model multiple times on the same dataset (Sinharay et al., 2001).

Another popular missing data approach is Full Information Maximum Likelihood (FIML), which is based on maximum likelihood estimation (Enders, 2001). In FIML, the likelihood that is maximized is composed of both the observed data and the conditional 
distribution of the missing data given the observed data. FIML produces consistent estimates as

long as the missing data mechanism is MCAR or MAR. Multiple Imputation and FIML will tend to produce similar results. However, choosing between either method mostly depends on software availability (Savalei and Rhemtulla, 2012).

\section{Aberrant Responses}

With the advent of technology and online data collection methods, aberrant responses have become an increasingly challenging and prevalent issue for researchers that rely on questionnaires (Chmielewski and Kucker, 2020). Aberrant responses are any type of response that may be suspect. Unlike missing data, there is no unifying framework proposed that encompasses all types of aberrant responses. Instead, there are different types of aberrant responses studied throughout the literature. For example, participants may provide untruthful responses due to social desirability. Other types of aberrant response patterns are caused by carelessness or inattentiveness (Meade and Craig, 2012). There is also insufficient effort responding where respondents are not fully motivated to give their best effort on questions (Huang et al., 2012; Curran 2016). An extreme form of insufficient effort responding is a survey bot, which is driven by automated survey completion programs to fill out surveys usually for monetary gains. Different underlying causes and manifestations of aberrant responses require different methodologies to detect them (Hong et al., 2020). There have also been a plethora of research papers on how to address responses contaminated by insufficient effort responding. Table 2 summarizes these various types of aberrant response methodology.

Table 2: Aberrant Response Methodology 
Type of Detection Description Example Methodology Method

\begin{tabular}{lll}
\hline Study Design & Methods that are planned & $\cdot$ Attention Checks \\
during the study design phase. & $\cdot$ Bogus Questions
\end{tabular}

Log Data

Statistical Methods
Methods that incorporate passive data collected during the study.

Methods that leverage statistical methods to detect respondents.
- Response Time

- Click Data

Note: This table was compiled based on works from Buchanan and Scofield (2018), Hong, Steedle and Cheng, (2020), and Meade and Craig, (2012).

Researchers have introduced many methods to detect aberrant responders. We introduce some of these methods here that fall into three broad categories. The first set of methods can be planned during the survey design phase and requires to be planned ahead of time. These methods include attention checks, instructed items, and other ways to evaluate a respondents attention during a survey (Meade and Craig, 2012). Researchers may also ask respondents if they should trust and use their data in their analysis at the end of the survey. What these methods have in common is that the researcher has to include these types of questions or checks in the survey in order to utilize these types of methods. If a researcher does not include these methods, then they have to rely on other approaches in order to detect aberrant responses.

Another way researchers can detect aberrant behavior is by analyzing log, or passive, data that is collected unobtrusively with a survey (Goodman et al., 2012). In these cases, log data can include response times, IP addresses, and other types of data researchers can utilize to detect 
aberrant responders (Meade and Craig, 2012). Note that these methods are restricted to data collected on a device, such as a computer or tablet. Others have used click count as a way to classify individuals as aberrant (Buchanan and Scofield, 2018). The advantage of using log data is that, most of the time, log data will be collected simultaneously with no additional cost with the survey responses. Another advantage of using log data is that aberrant responders may not be cognizant that these types of data will be collected, which strengthens the ability of the researcher to investigate how responders progress through a survey.

Finally, another class of methods one can use to detect or treat aberrant responses is by using statistical methods, ranging from a simple count of a long string of the same responses to complex statistical analyses that involve latent variable modeling. These types of approaches may require software and an understanding of the methodology. Statistical methods used to detect outliers or deviating patterns are studied throughout the literature, such as using mahalanobis distance (Hong et al., 2020) or person fit analysis based on item response theory (Patton et al., 2019). Moreover, there are other methods such as transforming the data to help mitigate the impact of aberrant responders (Yuan and Gomer, in press).

There have been previous meta studies that examined the methods researchers use to ensure quality data. Roth (1994) reviewed 45 articles between 1989 and 1991 and found that data removal was the most common approach for handling missing data. Peugh and Enders (1994) examined 989 samples from 1999 and 545 samples from 2003 about missing data practices. They found that few articles explicitly describe the missing data procedure and $96 \%$ of articles in 1999 employed either listwise or pairwise deletion. There was no mention of FIML or multiple imputation in any studies in 1999 and only 6 studies used either method in 2003. The authors 
were optimistic that researchers will begin to use more modern methods with the availability of more advanced software.

There were no meta studies to the authors' knowledge that have conducted a meta study about methodologies that investigate aberrant responders. However, there have been methodological studies that have investigated the efficacy of different methods to detect aberrant responders in different samples used in Psychology. Meade and Craig (2012) found that 10 to $12 \%$ of undergraduates completing a survey for course credit can be classified as careless responders. Similarly, Buchanan and Scofield (2018) found that about $14 \%$ of a sample from Amazon's Mechanical Turk can be considered aberrant using multiple methods as indicators of low data quality.

There are multiple choices a researcher can make when trying to minimize the impact of poor data quality induced by aberrant or missing responses. Given the recent interest in transparency in reporting psychological findings, we investigated what methodologies psychologists across different fields report to ensure good quality data in their studies regarding choices they made to address aberrant or missing responses. In the following section, we describe and report findings from a systematic review of psychological research regarding data preprocessing practices. We focus on reported methods and practices related to aberrant responses and missing data methods for self report (or informant report) measures. We then develop recommendations for data quality control in these contexts. Specifically, we aimed to answer the following questions:

1. What type of methodology do researchers use to handle missing or aberrant responses?

2. How often do authors report exclusion criteria in their studies due to poor quality data?

3. How much of a sample is inflicted by aberrant responses or missing data in a sample? 


\section{Method}

\section{Design}

This study collected a stratified sample based on the year and journal. Measured variables are shown in Table 1 . The study protocol was not preregistered. The anonymized data is available on the Open Science Framework (https://osf.io/ug4nz/).

\section{Sample}

We conducted our literature search within the Journal of Personality and Social Psychology (JPSP), Developmental Psychology, Abnormal Psychology, and Psychological Science. In order to obtain a representative sample, we conducted stratified sampling across the years 2012, 2015, and 2018. First, we assigned each article in the year to a random ID number and randomly sampled 20 articles within each journal and year. Our inclusion criteria included any article that contained a questionnaire. A questionnaire is a measure that contains at least one question with common item types, such as Likert items. We included both self report and informant reports in this study. If there were multiple studies within a manuscript, we included any study that contained a questionnaire and considered it one study. If there was data collected at multiple time points, we considered the entire data collection as one study and evaluated the quality of data at the final time point for the purpose of evaluating missing data. If a study did not meet our inclusion criteria, we continued sampling until our target sample size was met. Our final sample size was 240 articles and was chosen due to time and resources constraints.

\section{Coding Process}

We coded every article for several key variables related to data quality and preprocessing. Three of the authors coded each manuscript according to the outlined procedures. First, the coding process began where 20 articles were selected to be coded by the three authors. After 
identifying any discrepancies between each coder, the remainder of the articles were coded by two of the coders to ensure consistency. We did not calculate inter-rater reliability statistics because we reviewed and discussed each discrepancy. Any discrepancies were identified and discussed in a group on a case-by-case basis and our coding procedure was updated after each discrepancy. Table 3 presents all the final codes.

Table 3. Coded Variables

Variable Description Coded values

Type of Study Type of study refers to the data collection method. If a study used archival data or data from a database, we used available information from the database website.
Total Sample Size

Data Removal

Due to Missingness
The total sample size was determined by how much data was collected at the initial time point of the study. For a longitudinal study, the total sample size was considered the number of participants at the first time point.
- In-person

- Online

- Mixture of approaches

- Unclear about methodology

- Numeric Value
Data removal due to missingness refers to the total number of removed respondents due to missingness. If a study used an alternative method to data removal, such as Full Information Maximum Likelihood (FIML), we did not mark that the study lost data due to missingness.
- Numeric Value 
Data Removal Data removal due to aberrant response refers to the Due to Aberrant number of respondents a study removed due to Response suspicious behavior. If a study transformed the data or used robust methods, we did not mark that the study lost data.
Data Removal Due to Other Reasons

Data removal due to other reasons was recorded if a study removed data not due to missingness or potential aberrant responses. An example would be failing technology.

Missing Data Missing data methodology refers to how a study Methodology handled missing data.
- Numeric Value
- Numeric

Value
- Listwise deletion

- Full Information Maximum Likelihood

- Imputation
Aberrant
Aberrant response methodology refers to what Response methodology a study reported to detect or handle Methodology suspicious or aberrant responses.
- Ask Respondents
- Attention Check
- Extreme Values
- Following Instructions
- IP Address
- Log Data
- No Reason
- Random Responses
- Response Time
- Transform Value 
Preregistration For manuscripts published in 2018, we coded if a study had preregistered the study.

Incentives
Incentives refers to if a study mentions that participants were paid with monetary reward such as cash or a gift card. If a study mentioned a feebased web service, such as Amazon's Mechanical Turk, we assumed the respondents were paid.
- Registered

- Not registered

- Paid

- Not Paid

\section{Results}

\section{Descriptions of Studies}

Table 4 presents the sample characteristics of the 240 articles. Of the 240 coded studies, we found that $56.25 \%(135 / 240)$ were in-person studies, $20.83 \%(50 / 240)$ were online, $17.92 \%$ (43/240) were mixed, and 5\% (12/240) unspecified. 39.5\% (95/240) of the studies paid participants. Of the eighty studies published in 2018, $20 \%$ (16/80) preregistered their analysis. Across all studies, 39.58\% (95/240) paid their respondents. The median sample size was 530 (25 percentile $=195,75$ percentile $=1506)$.

Table 4: Sample Characteristics of 240 articles

Characteristic

\section{Journal}

Psychological Science

Developmental Psychology

Journal of Personality and Social Psychology

Abnormal Psychology
Number of Articles Percent of Articles

60

$25 \%$

60

$25 \%$

60

$25 \%$

60

$25 \%$ 
Year

2012

80

$33.33 \%$

2015

80

$33.33 \%$

2018

80

$33.33 \%$

\section{Data Collection Method}

In-person

Online

Mixed

Unclear

Preregistration in 2018

Yes

14

135

50

43

12

No

Payment

Yes

No
95

145
$5 \%$

$56.25 \%$

$20.83 \%$

$17.92 \%$

$17.5 \%$

$82.5 \%$

$39.58 \%$

$60.42 \%$

\section{Research Question 1: What type of methodology do researchers use to handle poor}

\section{data quality?}

Table 5 presents the missing data methodology reported in a manuscript. $46.25 \%$

(111/240) of studies reported not having any missing data and therefore reported no missing data approach. $29.58 \%$ (71/240) of articles conducted data removal (or listwise deletion). The remaining studies utilized a missing data method that did not require data removal, where the most popular approach was full information maximum likelihood. One study mentioned using Bayesian methods to handle missing data, but used vague language on how they handled 
missingness. Although missing data methods, such as multiple imputation, can be done in a Bayesian framework, the study did not explicitly describe the methodology used. Due to the ambiguity in the article, we coded it as a none reported.

Table 5: Missing Data Methodology

\begin{tabular}{lll} 
Missing Data Approach & Number of Articles & Percent of Articles \\
\hline Full Information Maximum Likelihood & 51 & $21.25 \%$ \\
Imputation & 8 & $3.33 \%$ \\
Listwise deletion & 71 & $29.58 \%$ \\
None reported & 111 & $46.25 \%$ \\
\hline
\end{tabular}

Note: One study used listwise deletion to remove data based on one variable and FIML with the remainder of the data.

Table 6 presents the aberrant response methodology reported in a manuscript. $80.83 \%$ (194/240) of studies reported not having any aberrant data and therefore reported no aberrant response approach. $7.08 \%$, (17/240) of articles removed aberrant data using statistical analysis, such as labelling certain respondents as extreme values. 5\% (12/240) of articles removed aberrant data because they failed attention checks. The remainder of the methodologies, such as using log data, was rare where less than $5 \%$ of the articles used these approaches.

Table 6: Aberrant Response Methodology

\begin{tabular}{ll} 
Aberrant Response Approach $\quad$ Number of Articles $\quad$ Percent of Articles \\
\hline
\end{tabular}

$\begin{array}{lcc}\text { Ask Respondents } & 1 & 0.41 \% \\ \text { Attention Check } & 12 & 5 \% \\ \text { Extreme Values } & 17 & 7.08 \%\end{array}$




$\begin{array}{llc}\text { Following Instructions } & 9 & 3.75 \% \\ \text { IP Address } & 4 & 1.67 \% \\ \text { Log Data } & 2 & 0.83 \% \\ \text { No reason removed } & 2 & 0.83 \% \\ \text { None reported } & 194 & 80.83 \% \\ \text { Random Responses } & 5 & 2.08 \% \\ \text { Response Time } & 2 & 0.83 \% \\ \text { Transform Values } & 2 & 0.83 \%\end{array}$

Note: 11 studies used multiple approaches to detect aberrant responses.

\section{Research Question 2: How often do authors report exclusion criteria due to missing} data and aberrant responses?

Across the 240 studies, 56.2\% (135/240) of studies do not report any exclusion criteria. $29.6 \%(71 / 240)$ of studies reported an exclusion criteria due to missingness. $17.5 \%(42 / 240)$ of studies reported an exclusion criteria due to aberrant responses. Figure 1 presents the reported exclusion criteria when conditioning on a variable of interest. Figure 1A presents the reported exclusion criteria by journal. Across journals, Developmental Psychology reports the fewest exclusion criteria where $78.33 \%$ (47/60) of the articles in the journal report utilizing all of the data. The journal that has the most studies excluding data due to missingness is JPSP with $40 \%$ (24/60) of studies. Similarly, JPSP had the most articles reporting exclusion criteria due to aberrant responders where $35 \%$ (21/60) of studies remove any data due to suspect responders.

Figure 1B presents the reported exclusion criteria by payment. If a study paid participants, it is more likely to report exclusion criteria due to aberrant responders (30/95; $31.58 \%)$ and missingness $(30 / 95 ; 31.58 \%)$. Studies that did not pay respondents had $8.28 \%$ 
$(12 / 145)$ of studies reporting exclusion criteria due to aberrant responders and $28.28 \%(41 / 145)$ of studies reporting exclusion criteria due to missingness.

Figure 1C presents reported exclusion criteria when conditioning on if a study in 2018 was preregistered. If a study preregistered their analysis, they were more likely to report exclusion criteria due to aberrant responders or missingness. Of the studies that preregistered their study, $42.86 \%(6 / 14)$ reported an exclusion criteria due to missingness compared to $24.24 \%$ $(16 / 66)$ when a study was not preregistered. Similarly, $35.71 \%(5 / 14)$ of articles that preregistered their study reported an exclusion criteria for aberrant responses compared to $12.12 \%(8 / 66)$ of articles that did not preregister their study.

Figure 1D presents the reported exclusion criteria by a study's data collection method. Studies employing in-person data collection were less likely to report any exclusion criteria for missing data $(35 / 135 ; 25.93 \%)$ or aberrant responders $(13 / 135 ; 9.63 \%)$. Mixed and online data collection methods were more likely to report an exclusion criteria for either missing data (online $17 / 50 ; 34 \%$, mixed $17 / 43 ; 39.53 \%$ ) or aberrant responders (online 12/50; 24\%, mixed 16/43; $37.21 \%)$.

Figure 1: Reported Exclusion Criteria 
A Reported Exclusion Criteria by Journal

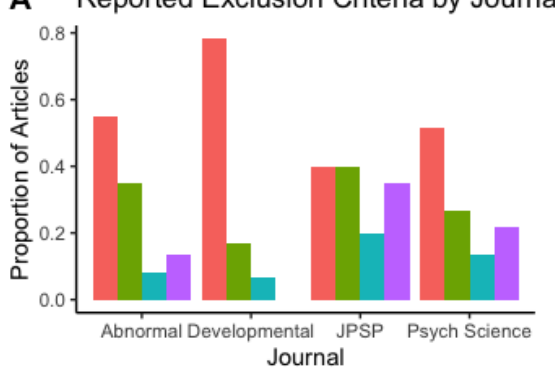

C Reported Exclusion Criteria by Preregister

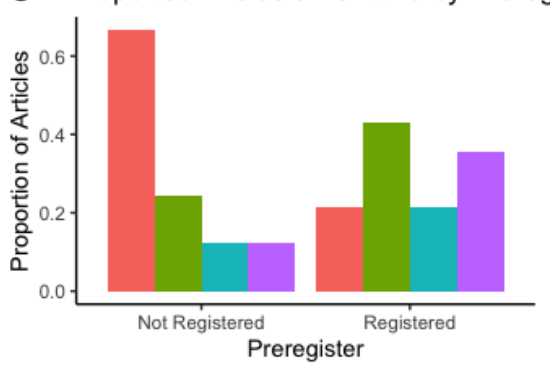

B Reported Exclusion Criteria by Payment

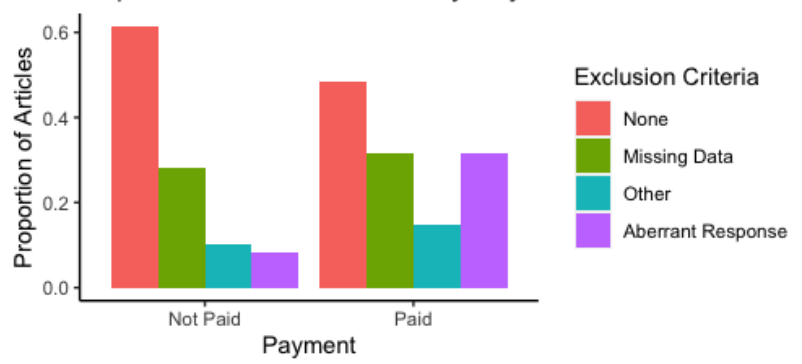

D Reported Exclusion Criteria by Data Collection Method

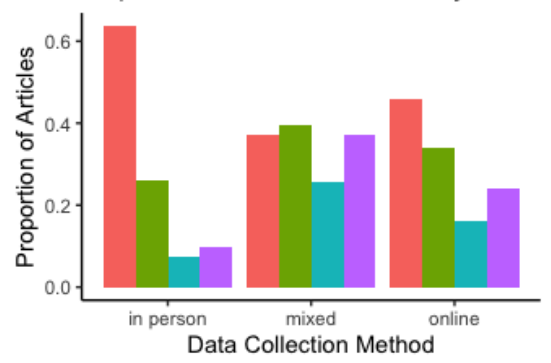

Exclusion Criteria

Exclusion Criteria

None

Missing Data

Other

Aberrant Response

Note: mixed refers to a mixture of online and in-person studies.

Research Question 3: How much of a sample is inflicted by aberrant responses or missing data in a sample and excluded from the analysis?

Given that a study removed data for a reason such as missing data or aberrant responses, we also calculated the proportion of the total sample size removed. If a study used FIML or multiple imputation, the missing data was not considered excluded. Aberrant responses were not considered excluded if a study used robust methods or transformed their data.

Across all studies that excluded data, $12 \%$ of a sample was removed due to missingness and $4 \%$ of a sample was removed as an aberrant response. Figure 2 presents the proportion of the sample removed when conditioning on different variables of interest. Figure 2A presents the proportion of a sample removed by studies appearing in different journals. Abnormal Psychology excluded the largest proportion $(11 \%)$ of the sample due to aberrant responses. Developmental Psychology did not exclude any respondents that were suspected to be aberrant. However, Developmental Psychology did exclude the largest proportion of the sample due to missing data 
(20\%), possibly due to a larger presence of longitudinal studies in developmental research. JPSP had the smallest proportion of a sample excluded due to missingness (7\%).

Figure $2 \mathrm{~B}$ presents the proportion of a sample removed by study payment. There appears to be more missing data removed when respondents are not paid (15\%) compared to when they are paid $(9 \%)$. There is no difference in the number of aberrant responders if one chooses to pay respondents as $4 \%$ of the sample is excluded as aberrant responders in both cases.

Figure $2 \mathrm{C}$ presents the proportion of a sample removed by preregistration status. There were no differences between preregistered and nonpreregisted studies in the proportion of a sample excluded to missingness where only $12 \%$ of the sample was excluded due to missingness. Similarly, there were small differences in the proportion of a sample excluded due to aberrant responders where $3 \%$ or $5 \%$ of a sample is removed given a study is preregistered or not, respectively.

Figure 2D presents the proportion of a sample removed by data collection method. Inperson data collection had the largest proportion of the sample removed due to either aberrant responses $(6 \%)$ or missingness $(16 \%)$. Mixed data collection approaches were associated with smaller proportions of the sample removed due to either missingness $(6 \%)$ and aberrant responses $(4 \%)$.

Figure 2: Proportion of the Sample Removed 

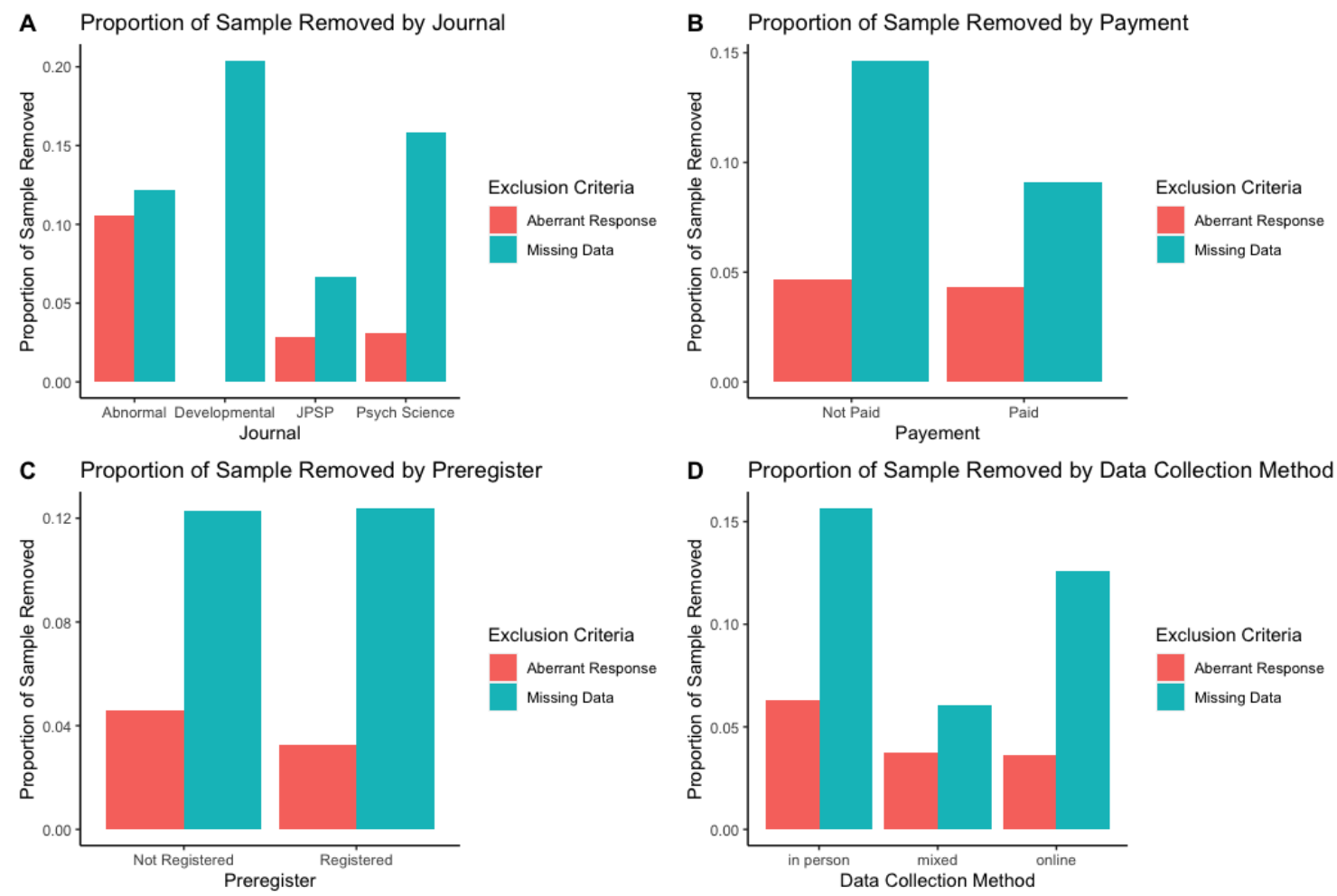

\section{Discussion}

Ensuring good data quality is an integral component of the research process. In this article, we focus on data quality and preprocessing related to aberrant responses and missing data. Problems with data quality have been recently called for especially with the large scale data collection methods on platforms such as Amazon's Mechanical Turk (Buchanan and Scofield, 2018; Chmielewski and Kucker, 2019). Without high quality data, the validity of research findings will be compromised. Our findings suggest that researchers tend to underreport the data preprocessing steps in their studies in the context of missing data or aberrant responses.

Moreover, many researchers rely only on suboptimal procedures. Furthermore, flexibility in the steps or choices in data preprocessing may hinder the replicability, or even reproducibility, of findings from studies and contribute to the so-called replication crisis.

Transparency in Reporting Data Quality and Preprocessing Methods 
There has been an interest in psychology for more transparency of methodology. However, we found that a substantial portion $(111 / 240 ; 46.25 \%)$ of studies do not report any missing data handling methodology. This finding is comparable to other meta research on the same topic. In 1999, only $34 \%$ of studies explicitly address missing data issues in the main manuscript (Peugh and Enders, 2004). The proportion of studies that do not mention a plan or procedure for aberrant responses is even larger, where many $(194 / 240 ; 80.83 \%)$ studies do not report any method for exclusion criteria for aberrant responses.

We explored this finding further by investigating more recent practices in 2018 with a focus on preregistration. In theory, preregistered studies should be more transparent in their reporting of methodology. Indeed, most preregistration templates recommend explicitly mentioning data exclusion criteria (Nosek et al., 2018). In the current study, there were 14 preregistered studies and 66 non-preregistered studies in 2018. A preregistered study is more likely to report their aberrant and missing data handling methodology relative to non preregistered studies. One would hope that all preregistered studies would report their data quality methodology. Although a study should not need to report their data preprocessing methodology in the main manuscript because it is in the preregistration, other researchers have found that several studies deviate from their original analysis in a plethora of ways (Claesen et al., 2019). In a recent review of articles in Psychological Science, researchers found 56\% of studies had undisclosed deviations with regards to exclusion criteria. The authors argue that without explicit mentions in the preregistration and final manuscript, there may be discrepancies that arise between the final manuscript and preregistered study. One or more planned exclusion criteria may not have been used or some exclusion criteria were not included in the original 
preregistration. Therefore, explicit details concerning data preprocessing in a manuscript are warranted in order to evaluate the scientific claims of a study.

Moreover, data preprocessing is inherently data dependent even if one has a substantive theory to guide their analysis plan. Consider the so-called garden of forking paths (Gelman and Loken, 2013). A dataset can be analyzed using various data quality control methods. It can be easy to find a statistically significant result if one considers multiple ways to analyze the data. In Simmons, Nelson and Simonsohn (2011) the authors suggest that if observations are removed, then they should report the statistical results with and without those observations. The veracity of a researcher's claims can only be evaluated by the consumers if the final data preprocessing methodology and analysis are reported in the manuscript.

\section{Missing Data Methodology}

This study also identified issues pertaining to data quality and preprocessing methodology throughout the literature. We first consider the issues with data preprocessing with regards to missing data. If a study does report missing data methodology, the most common methodology is listwise deletion $(71 / 129 ; 55.04 \%)$. The second most popular approach was FIML $(51 / 129 ; 39.53 \%)$. Only a few studies used any form of imputation $(8 / 129 ; 6.2 \%)$. In the context of missing data, this is problematic for a few reasons. Listwise deletion is a suboptimal missing data procedure for most cases as outlined earlier. Listwise deletion requires the missing data mechanism to be MCAR. This means the missingness does not depend on either observed or missing variables, which is highly unlikely. Furthermore, researchers will have a loss of power because some of the data are being removed from the analysis. There are methods that can subsume the MCAR assumptions without removing any data, such as FIML or imputation. Furthermore, our review suggests that, on average, $12 \%$ of a sample would be removed due to 
missingness. This finding suggests that previous research findings may be biased based on the methodological literature on missing data (Enders and Bandalos, 2001). However, certain study designs, such as longitudinal studies, are more likely to employ advanced methods to handle missing data. This finding was supported where the journal Developmental Psychology had no studies removing any data due to missingness. As noted in most developmental journals, missing data is the rule rather than the exception in longitudinal studies. As a field, developmental psychology appears to embrace advanced missing data treatment methodology.

However, one thing that was neglected throughout all the studies is that no study entertained the possibility of MNAR. Although this is a difficult problem to determine, it would be worthwhile for studies to consider what data they collect and how they collect it with respect to missing mechanisms. If a researcher suspects that they will have missingness, and hopes to inform other researchers on the missing data mechanism, then a study could report how they addressed missingness. This means reporting auxiliary variables, or variables within the original data that are not included in the analysis but may be related to variables of interest or involved with the missing data process (Little, 1992).

\section{Aberrant Responses Methodology}

We found that most research $(194 / 240 ; 80.83 \%)$ did not report any exclusion criteria for aberrant responses. Common quality control methods used were survey design features such as attention checks $(12 / 46 ; 26.09 \%)$ and identifying respondents who failed to follow instructions $(9 / 46 ; 19.57 \%)$. Furthermore, some studies used statistical methods to remove data because they were considered extreme values $(17 / 46 ; 36.96 \%)$. Only a few studies used other methods, such as response times or log data, to identify aberrant responses. All studies in the journal Developmental Psychology, where most studies leverage longitudinal designs, do not report any 
methodology to identify suspect responses. This suggests that studies that using complex sampling designs may ignore the possibility of aberrant responses.

On average, a study removed $4 \%$ of the data that was labelled as aberrant. However, the true amount of aberrant data remains unclear as more than three quarters of the studies do not report any exclusion criteria for aberrant responses. Furthermore, it is often recommended to use multiple methods to detect different types of aberrant responders, but only a few $(11 / 240 ; 4.58 \%)$ studies used more than one method. For these reasons, one can suspect that many studies have more aberrant responses hidden in the dataset. Even with the low reported aberrancy level, studies may have reported biased statistics, such as a correlation coefficient, due to ignoring aberrant responses. If one hopes to achieve an accurate representation of a phenomena, researchers need to consider the possibility of aberrant responses in their data and consider reporting their methodology to detect aberrant responders so their findings can be replicated.

\section{Respondent Payment}

Another factor when assessing the quality of data is whether or not the respondents were paid. There has been interest whether data quality control improves if one provides sufficient funds for the time of the respondent (Downs et al., 2010; Paolacci et al., 2010). Some researchers found that payment was not a main motivating factor when completing surveys or tasks (Mason et al., 2012).

The current findings provide new light where there is, on average, more missing data in a sample when respondents are not paid (15\%) compared to when they are paid (9\%). This finding suggests paying respondents may elicit more responses with a survey. However, paying respondents does not change the proportion of engaged respondents in a sample, where there remains about $4 \%$ of respondents considered aberrant in both groups. This suggests that if 
researchers hope to minimize the impact of missing data, one should consider paying respondents; but to discourage aberrant response behavior, other strategies may still be necessary.

\section{In-person and Online Data Collection}

The quality of data may also be affected by the mode in which the respondent took the survey. Indeed, some researchers question the validity of scores collected online as the anonymity of respondents may induce carelessness or inattentive behavior (Buchanan, 2000). Others have argued that internet samples provide comparable psychometric properties of scales such as the Big Five (Gosling et al., 2000).

In the current study, in-person data collection had the largest proportion of the sample removed due to either aberrant responses $(6 \%)$ or missingness $(16 \%)$. Online studies removed only $4 \%$ of the respondents due to aberrant responses and $13 \%$ due to missingness. The current findings suggest that online studies may provide better quality data relative to in-person studies. There is no evidence that collecting data online provides obviously worse quality data compared to in-person data collection.

Researchers who conducted online data collection were more likely to utilize any data preprocessing method for missing data and aberrant responses $(27 / 50 ; 54 \%)$ compared to online studies $(49 / 135 ; 36.29 \%)$. This suggests that online studies may elicit a heightened awareness of data quality control concerns.

\section{Recommendations}

Our review suggests that data quality concerns are important across subareas of psychology and highlight the role of ensuring good data quality and transparency to provide valid and replicable inferences. The practice of reporting evidence of good data quality is 
imperative. We recommend several resources to ensure good data quality for researchers in Tables 1 and 2. In summary, we recommend the following:

1. Explicitly report the final used exclusion criteria in a study, even if the material is found in a preregistration.

2. Consider alternative missing data handling practices beyond listwise deletion, such as FIML and imputation.

3. Plan for different missing data scenarios and collect data that may be related to the missing data mechanism.

4. Incorporate one or more methodologies to detect aberrant responders in a study.

5. To conduct a sensitivity analysis when leveraging different strategies to improve data quality.

6. To pay respondents for their time to improve survey completion.

We hope the present assessment and recommendations may serve as a starting point for improving the data quality in psychology. 


\section{References}

Buchanan, T. (2000). Potential of the Internet for Personality Research. In Psychological Experiments on the Internet. https://doi.org/10.1016/b978-012099980-4/50006-X

Buchanan, E. M., \& Scofield, J. E. (2018). Methods to detect low quality data and its implication for psychological research. Behavior Research Methods. https://doi.org/10.3758/s13428-018-1035-6

Chmielewski, M., \& Kucker, S. C. (2020). An MTurk Crisis? Shifts in Data Quality and the Impact on Study Results. Social Psychological and Personality Science. https://doi.org/10.1177/1948550619875149

Claesen, A., Gomes, S. L. B. T., Tuerlinckx, F., \& Vanpaemel, W. (2019). Preregistration: Comparing Dream to Reality. PsyArXiv Preprints. https://doi.org/10.31234/osf.io/d8wex

Curran, P. G. (2016). Methods for the detection of carelessly invalid responses in survey data. Journal of Experimental Social Psychology. https://doi.org/10.1016/j.jesp.2015.07.006

Downs, J. S., Holbrook, M. B., Sheng, S., \& Cranor, L. F. (2010). Are your participants gaming the system? Screening mechanical Turk workers. In Conference on Human Factors in Computing Systems - Proceedings. https://doi.org/10.1145/1753326.1753688

Enders, C. K. (2001). The performance of the full information maximum likelihood estimator in multiple regression models with missing data. Educational and Psychological Measurement. https://doi.org/10.1177/00131640121971482

Enders, C. K., \& Bandalos, D. L. (2001). The relative performance of full information maximum likelihood estimation for missing data in structural equation models. Structural Equation Modeling. https://doi.org/10.1207/S15328007SEM0803_5

Enders, C. K. (2010). Applied missing data analysis. Guilford Press.

Gelman, A., \& Loken, E. (2013). The garden of forking paths. https://doi.org//S071610182009000400017

Gomer, B., \& Yuan, K. H. (in press). Subtypes of the Missing Not at Random Missing Data Mechanism. Psychological Methods. 
Goodman, J. K., Cryder, C. E., \& Cheema, A. (2012). Methodological Concerns and Solutions in Mechanical Turk Experimentation. Consumer Research.

Gosling, S. D., Vazire, S., Srivastava, S., \& John, O. P. (2000). Should we trust web-based studies? American Psychologist.

Hong, M., Steedle, J. T., \& Cheng, Y. (2020). Methods of Detecting Insufficient Effort Responding: Comparisons and Practical Recommendations. Educational and Psychological Measurement. https://doi.org/10.1177/0013164419865316

Huang, J. L., Curran, P. G., Keeney, J., Poposki, E. M., \& DeShon, R. P. (2012). Detecting and Deterring Insufficient Effort Responding to Surveys. Journal of Business and Psychology. https://doi.org/10.1007/s10869-011-9231-8

Little, R. J. A. (1992). Regression with missing X's: A review. Journal of the American Statistical Association. https://doi.org/10.1080/01621459.1992.10476282

Little, R. J. A., \& Rubin, D. B. (2019). Statistical analysis with missing data. Statistical Analysis with Missing Data. https://doi.org/10.1002/9781119482260

Mason, W., \& Suri, S. (2012). Conducting behavioral research on Amazon's Mechanical Turk. Behavior Research Methods. https://doi.org/10.3758/s 13428-011-0124-6

Meade, A. W., \& Craig, S. B. (2012). Identifying careless responses in survey data. Psychological Methods, 17(3), 437-455. https://doi.org/10.1037/a0028085

Nosek, B. A., Ebersole, C. R., DeHaven, A. C., \& Mellor, D. T. (2018). The preregistration revolution. Proceedings of the National Academy of Sciences of the United States of America. https://doi.org/10.1073/pnas.1708274114

Patton J., Cheng Y., Hong M., Diao Q. (2019). Detection and Treatment of Careless Responses to Improve Item Parameter Estimation. Journal of Educational and Behavioral Statistics.. doi:10.3102/1076998618825116

Paolacci, G., Chandler, J., \& Ipeirotis, P. G. (2010). Running experiments on Amazon mechanical turk. Judgment and Decision Making.

Peugh, J. L., \& Enders, C. K. (2004). Missing data in educational research: A review of reporting practices and suggestions for improvement. Review of Educational Research. https://doi.org/10.3102/00346543074004525

Roth, P. L. (1994). Missing Data: a conceptual review for applied psychologists. Personnel Psychology. https://doi.org/10.1111/j.1744-6570.1994.tb01736.x 
Rubin, D. B. (1976). Inference and missing data. Biometrika. https://doi.org/10.1093/biomet/63.3.581

Savalei, V., \& Rhemtulla, M. (2012). On Obtaining Estimates of the Fraction of Missing Information From Full Information Maximum Likelihood. Structural Equation Modeling. https://doi.org/10.1080/10705511.2012.687669

Simmons, J. P., Nelson, L. D., \& Simonsohn, U. (2011). False-Positive Psychology. Psychological Science, 22(11), 1359-1366. https://doi.org/10.1177/0956797611417632

Sinharay, S., Stern, H. S., \& Russell, D. (2001). The use of multiple imputation for the analysis of missing data. Psychological Methods. https://doi.org/10.1037/1082989x.6.4.317

Yuan, K. H., \& Gomer, B. (2021). An overview of applied robust methods. British Journal of Mathematical and Statistical Psychology. https://doi.org/10.1111/bmsp.12230 